- Prevention is a fundamental element of clinical practice. This is the first in a series of papers which will review and update the evidence base for preventive action in general dental practice.

- One important, but underdeveloped area of prevention is smoking cessation. Smoking and tobacco use adversely affects oral health in a variety of ways. The dental profession and their teams have a potentially important role to play in helping smokers to quit.

- This paper aims to review the practical steps that the dental team can implement in smoking cessation activities within the clinical dental setting.

\title{
Prevention. Part 1: Smoking cessation advice within the general dental practice
}

\author{
R. G. Watt ${ }^{1}$ and B. Daly ${ }^{2}$; Series Editor E. J. Kay ${ }^{3}$
}

\begin{abstract}
Smoking remains the largest single preventable cause of death and disability in the UK and costs the NHS $£ 1.7$ billion each year. ${ }^{1}$ More than 120,000 people die prematurely due to smoking related diseases. Worldwide smoking is the single most important public health problem. The detrimental effects of smoking and tobacco use on oral health are well recognised. Oral cancers and pre-cancers, periodontal diseases and poor wound healing are the most significant and serious effects of smoking on the mouth. ${ }^{2,3}$ In addition, staining of the teeth, soft tissue changes and halitosis are aesthetic and social impacts of smoking directly related to oral health.
\end{abstract}

\section{PREVENTION}

1. Smoking cessation advice

2. Dietary advice

3. Prevention of tooth wear

4. Toothbrushing advice

5. Patients requiring osseointegrated oral implant treatment

6. Older dentate patient

7. Professionally applied topical fluorides for caries prevention

8. Pit and fissure sealants in preventing caries in the permanent dentition of children

1*Reader, Department of Epidemiology and Public Health, Royal Free and University College London Medical School, University College London, 1-19 Torrington Place, London WC1E 6BT; ${ }^{2}$ Lecturer, Department of Dental Public Health, The Guy's, Kings and St. Thomas' Schools of Medicine, Dentistry and Biomedical Sciences. GKT Dental Institute, Caldecot Road, London SE5 9RW

${ }^{3}$ Professor of Dental Health Services

Research, University of Manchester Dental Hospital and School, Higher Cambridge

Street, Manchester M15 6FH

*Correspondence to: $\mathrm{R} \mathrm{G}$ Watt

E-mail:r.watt@ucl.ac.uk

\section{Refereed Paper}

(c) British Dental Journal 2003; 194

665-668
Recently published evidence-based guidelines on smoking cessation have highlighted the important role that health professionals can play in helping smokers to stop successfully. ${ }^{4}$ Systematic reviews of many randomised controlled trials have shown the effectiveness of smoking cessation advice provided by general medical practitioners. On the recognised hierarchy of evidence, these would be classified as Type 1 evidence. Relatively few well designed studies have been undertaken to assess the effectiveness of dental professionals in smoking cessation activities although the success rates achieved are comparable with studies in other primary care settings. ${ }^{5,6}$ Indeed factors such as access to smokers, level of training, experience and commitment are more important in determining success than professional discipline. ${ }^{7}$

Although many dentists express positive attitudes to becoming actively involved in smoking cessation activities with their patients, few routinely assist smokers to stop. ${ }^{8,9}$ This paper aims to outline ways in which dental practitioners and their teams can support smokers to effectively quit. Very limited time is required when assistance and support is provided in a standardised way to those smokers interested and willing to stop. Involvement in smoking cessation provides an opportunity for dentists to become engaged in an interesting, relevant and important area of prevention.

\section{WHO SMOKES ANYMORE?}

In the UK around $28 \%$ of the adult population smoke, that is over 13 million smokers. ${ }^{10}$
Although the overall rate of smoking has declined steadily since the 1940 s, smoking is now increasingly restricted to the more disadvantaged sections of society. For example, in 1996, 12\% of men in professional occupations smoked compared with $40 \%$ of men in unskilled manual jobs. ${ }^{11}$ Although dental attendance is inversely related to deprivation, many smokers will be seen by general dental practitioners.

Across many parts of the developed world concern has been expressed about the continuing problem of teenage smokers, especially amongst young women. The vast majority of adult smokers start during their adolescence. Once started, although many smokers report a desire to give up, they will become addicted to nicotine and will spend years struggling to break the habit

\section{TIME FOR ACTION}

Based upon a systematic review of the scientific evidence, a smoking cessation protocol has been published and updated to encourage health professionals, including dentists to become more actively involved in smoking prevention. ${ }^{4,11}$ The 4 A's model is a straightforward and quick means of identifying smokers who want to stop and how best to help them achieve their goal (Fig. 1).

Advice and support provided in a clinical setting will be most effective with patients who are interested and keen to make a quit attempt. Pressurising 'contented' smokers will most often achieve very little. It is essential to tailor advice and support to smokers who are ready and willing to change their behaviour. The use of appro- 
Table 1 Questioning techniques and styles

- Use both closed and open questions, as appropriate, depending on what information you require

- Give patients time to answe your questions - don't rush them!

- Encourage patients to speak openly and honestly

- Take care not to be seen as nagging or judgemental - this achieves nothing

- Sum up any information given to you to check you have understood what has been said

\section{Heirarchy of evidence}

\section{Type 1 \\ Systematic review of at least one randomised controlled trial (RCT) \\ Iype 2 \\ At least one RCT \\ Iype 3 \\ Non-randomised intervention studies \\ Type 4 \\ Observational studies \\ Iype 5 \\ Traditional reviews, expert opinion}

priate questioning techniques is therefore very important (Table 1).

The different steps in the 4 A's model will now be outlined.

\section{Ask}

All patients should have their smoking status checked at the start of each course of treatment. A simple and quick system should be devised to record smoking details in the patient's clinical notes. This information should be kept up to date as possible. The following questions can be used within a standard medical history to assess whether the patient smokes, their level of nicotine addiction and their motivation to stop.

\section{Are you a smoker?}

How many cigarettes do you smoke each day? How soon after waking up in the morning do you have your first cigarette?

Have you ever tried to stop smoking?

Are you interested in stopping now?

Patients who report smoking more than 20 cigarettes per day and who have their first cigarette within 30 minutes of waking up in the morning are likely to be heavily dependent upon nicotine and will require more specialised and intensive support. These individuals are best referred to the local specialist services for help.

\section{Advise}

All smokers and those using other forms of tobacco should be advised of the value of stopping. The advice should be clear, firm and personally relevant. Although most people are aware of the harmful effects of smoking in relation to lung cancer and heart disease, fewer people know about the detrimental effects of smoking on their oral health. ${ }^{12}$ This provides a unique opportunity for dentists and members of the team to highlight the dangers of smoking in what could be considered an appropriate setting, the dental surgery. Scarring patients with frightening images of diseased organs may not be effective for many people. Instead a range of reasons for stopping smoking could be highlighted, some directly related to oral health, others more general (Table 2). Consider what is likely to be most significant and relevant to the patient. For example, stained teeth, halitosis and soft tissue changes in the mouth may be especially pertinent to young people. The early effects of tobacco use on the mouth are visible and reversible and may be a useful means of motivating smokers on the benefits of stopping. All smokers however will have their own good reasons for stopping.

\section{Assist}

If during the first two stages a smoker expresses a desire to quit, help and support should be offered. For those smokers not ready or willing to give up at this point, it is best raising the issue

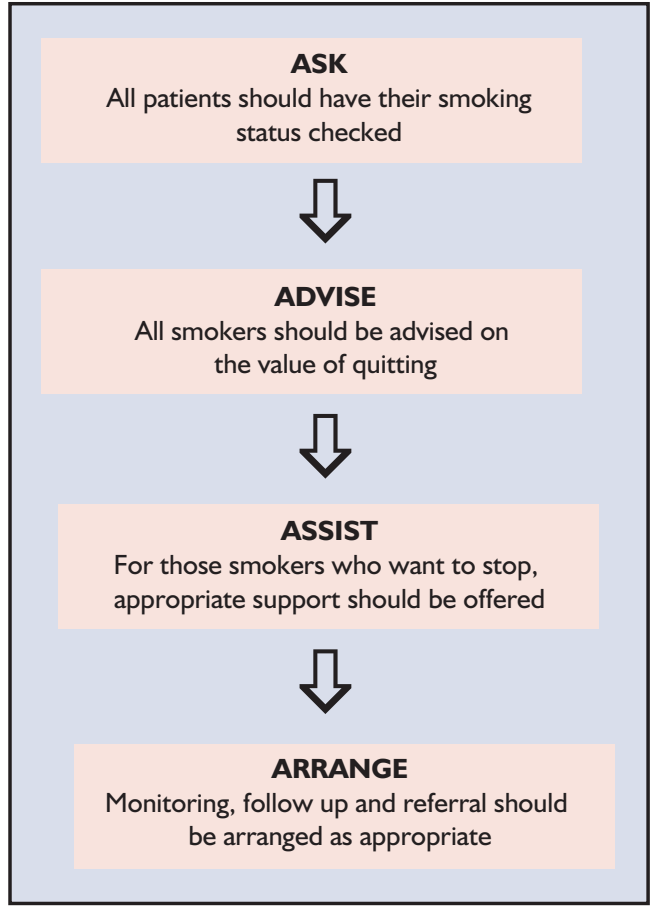

Fig. 1 The four A's approach to smoking cessation

again at a later stage to check if they have changed their opinions. Putting pressure or nagging smokers who are not ready to quit can be counterproductive and a waste of your and their time.

Assistance for those wishing to quit should focus on the following points:

- Negotiate a quit date - smokers need time to prepare

- Review past experiences of quitting - identify what helped and hindered progress in the past

- Identify any potential problems ahead and plan how these can be dealt with

- Stress the importance of enlisting the support of friends, family and colleagues - their assistance is essential

- Explore the value of using nicotine replacement therapy and Bupropion (Zyban) (see below for further details)

- Give details of telephone support lines which can provide on going support and encouragement (see the box on the adjacent page for a list of useful phone numbers)

\section{Arrange}

Monitoring progress is an essential part of successful cessation. Arranging a follow up is therefore very important. Evidence indicates that ideally patients should be initially seen 1-2 weeks after their quit date. This may fit in with a subsequent dental appointment for on going treatment or a visit to the hygienist. At this early stage people need support and encouragement. Congratulate patients who have managed not to smoke over this period. Praise and encouragement can help motivate and maintain patients determination to succeed.

Those patients who have smoked since their 
Table 2 Potential reasons for quitting smoking

- Reduce risk of halitosis

- Improve appearance - less staining on teeth, better skin texture

- Save money

- Feel better and more energetic

- Break dependence on tobacco

- Reduced risk of cancers and heart disease

- Better periodontal health - greater chance of retaining teeth for life

- Reduced risk of oral cancers

- Improved success with surgical treatments

quit date need your support and encouragement too. Most smokers make several attempts to stop before finally succeeding. Through sensitive questioning it is important to find out what happened and any lessons that can be drawn from the experience. This will help them in future attempts to predict possible problems and increase their confidence to succeed.

Some smokers who are heavily dependent on nicotine may require more specialist and intensive support to quit. Across the country specialist smoking cessation services have been established to provide assistance to these individuals. Dentists can refer patients to these services, details of which should be available with the local Primary Care Trust or Health Promotion Service.

\section{IMPORTANT AIDS TO SUCCESS}

A range of factors make quitting smoking a difficult task for many people. One of the most important problems is the powerfully addictive nature of nicotine. Nicotine replacement therapy (NRT) can help people cope better with their cravings for nicotine, particularly moderate smokers. The use of NRT doubles a persons cessation success rate. A range of NRT products are available including patches, gums, nasal sprays, inhalators and microtabs. Recently a NRT lozenge product has also been launched. The choice of product largely depends on personal preference. NRT products have recently been made available on prescription which has helped improve access by reducing their cost, which was previously a significant barrier to their use especially amongst low income smokers.

Bupropion (Zyban), although originally developed as an anti-depressant in the US has now been licensed as a pharmaceutical treatment for tobacco dependence. A meta analysis of published trials demonstrate that the drug improves 12 month abstinence rates and reduces the severity of withdrawal symptoms. ${ }^{13}$ The drug is available on prescription through GPs and specialist smoking clinics.

\section{SMOKING CESSATION WORKS}

The evidence base demonstrating the value of smoking cessation in primary care settings is very strong, based upon the findings of a number of randomised clinical trials. ${ }^{4}$ Very brief advice lasting less than 3 minutes given by a health professional will help an additional $2 \%$ of smokers to successfully stop smoking each year. With more intensive support lasting up to 10 minutes, plus NRT, an additional 6\% of smokers will quit.

A quit rate of $2 \%$ or even $6 \%$ may seem rather insignificant. However when translated into a population estimate, between 63,000 and 190,000 people may quit smoking each year in the UK if all general dental practitioners routinely offered smoking cessation advice based on the 4 A's model to those seeking dental care. ${ }^{11}$ Such an impressive achievement for the dental profession can be achieved through the routine adoption of a straightforward and relatively quick protocol.

\section{MOVING THE AGENDA FORWARDS}

A range of barriers have been reported by dentists to explain the low level of routine involvement in smoking cessation activities within dental practices despite positive attitudes. ${ }^{6,9,14-16}$ The main barriers include time and cost pressures, lack of knowledge and confidence, concerns over impact on dentistpatient relationship, doubts of the effectiveness of interventions and lack of resources for use in dental settings. How can these barriers be overcome? Table 3 outlines a variety of factors to facilitate future action.

Table 3 Facilitators for action with smoking cessation

- Reimbursement - introduction of smoking cessation fee item into GDS payment schedule

- Professional development - expansion of training opportunities in a range of topics from communication skills to knowledge of smoking treatments

- Team working - delegation of roles and responsibilities and development of team approach

- Improved communication - between staff on roles and responsibilities, with patients on relevance of oral health and smoking, and with history taking and clinical note keeping

- Multi-disciplinary working - referral of cases to specialist support when required

- Resource development - need for relevant materials for history taking and support materials for patients

- Research - need for improved evidence base on effectiveness of smoking cessation within dental settings

\section{CONCLUSION}

Smoking is the single most important public health challenge facing the NHS. The continued toll of suffering, disease and premature death resulting from tobacco use requires effective and concerted action. The government launched a national anti-smoking strategy in 1998 to co-ordinate action across the health service and beyond. ${ }^{17}$ Dental professionals have been identified as having an important role to play in supporting smokers who desire to quit. Evidence- based guidelines provide a clear way forward for all health professionals to become engaged in this important area of prevention. ${ }^{4,7}$ A reduction in smoking levels would improve both general and oral health,
Between 63,000 and 190,000 people may quit smoking each year in the UK if all GDPs routinely offered smoking cessation advice

Telephone support lines

Quitline 0800002200

NHS Smoking

Helpline

08001690169

NHS Pregnancy Smoking Helpline 08001699169 
and would help to reduce widening inequalities across the population.

\section{RECOMMENDATIONS ON SMOKING CESSATION WITHIN DENTAL SURGERIES}

1. Tobacco use is a recognised risk factor for a host of conditions including a range of oral diseases (Type 1 Evidence).

2. Dental professionals should establish the smoking status of their patients on a regular basis (Type 3 Evidence).

3. Dental professionals should advise all smokers to stop and emphasise the oral health benefits of quitting (Type 3 Evidence).

4. Smokers who are interested and motivated to quit should be given appropriate assistance by dental professionals (Type 3 Evidence).

5. Smokers attempting to quit should be monitored and supported. Dental professionals should refer heavy smokers or those with complex needs to specialist smoking cessation services (Type 3 Evidence).

1. Callum C. The UKsmoking epidemic: Deaths in 1995. London: Health Education Authority, 1998.

2. Legarth J, Reibel J. EU Working Group on Tobacco and Oral Health. Oral Dis 1998; 4: 48-67.

3. Johnson N W, Bain C. Tobacco and oral disease. Br Dent 2000; 4: 200-206

4. Raw M, McNeill A, West R. Smoking cessation guidelines for health professionals. A guide to effective smoking cessation interventions for the health care system. Thorax 1998; Suppl. 5: 1-38.
5. Cohen SJ Stookey G K Katz B P. Drook CA Christen A G Helping smokers quit: a randomised controlled trial with private practice dentists. JADA 1989; 118: 41-45.

6. Smith SE, Warnakulasuriya KA A S, Feyerabend C, Belcher M, Cooper D J, Johnson N W. A smoking cessation programme conducted through dental practices in the UK. BrDent J 1998; 185: 299-303.

7. West $R$, McNeil A, Raw M. Smoking cessation guidelines for health professionals: an update. Thorax 2000; 55: 987-999.

8. John J H, Yudkin P, Murphy M, Ziebland S, Fowler G H. Smoking cessation interventions for dental patientsattitudes and practices of dentists in the 0 xford region. $\mathrm{Br}$ Dent J 1997; 183: 359-364

9. McCann M, Macpherson L M D, Binnie V etal.A survey of Scottish primary care dental practitioners' oral cancerrelated practices and training requirements. Community Dent Health 2000; 17: 24-30

10. Thomas M, Walker A, Wilmot A, Bennet N. Office for National Statistics. Living in Britain: results from the 1996 General Household Survey. London: The Stationery Office, 1998.

11. Watt R, Robinson M. Helping smokers to stop - a guide for the dental team. London: Health Education Authority, 1999.

12. Warnakulasuriya K, Harris C, Scarrott D et al. An alarming lack of public awareness towards oral cancer. Br Dent J 1999; 187: 319-322.

13. Jorenby D, Leischow S, Nides M et al. A controlled trial of sustained release bupropion, a nicotine patch, or both for smoking cessation. NEng J Med 1999; 340: 685-691.

14. Chestnutt I G, Binnie V I. Smoking cessation counselling-a role for the dental profession? Br Dent J1995: 179: 411-415.

15. Warnakulasuriya KAAS, Johnson N W. Dentists and oral cancer prevention in the UK: opinions, attitudes and practices of screening for mucosal lesions and to counselling patients on tobacco and alcohol use: baseline data from 1991. Oral Dis 1999; 5: 10-14

16. Allard R H B. Tobacco and oral health: attitudes and opinions of EU dentists; a report of the EU working group on tobacco and oral health. Int Dent J 2000; 50: 99-102.

17. Smoking Kills: A White Paper on Tobacco. London: The Stationery Office, 1998. 